Aus der kgl. dermatologischen Universitätsklinjk in Breslau.

(Stellvertretender Direktor: Privatdozent Dr. Zi eler.)

\title{
Zur Behandlung der Syphilis mit Mergal,
}

\section{nebst quantitativen Untersuchen über die dabei eintretende Hg-Ausscheidung durch die Nieren.}

Von

\author{
Dr. Fritz Höhne,
}

früherem Assistenten đer Klinitik.

(Hiezu Taf. XV.)

Die interne Quecksilberanwendung ist seit langer Zeit besonders in Frankreich als bequemste Methode der Syphilisbehandlung viel geübt worden, hat sich aber sonst nur wenig Anhänger erwerben können. Welander1) sieht sie als so unsicher an, daß er sie seit mehreren Jahren nur noch ganz ausnahmsweise anwendet. Neisser sagt: "Wenn auch nachgewiesen worden ist, daß per os eingeführte Quecksilberpräparate regelmäßig mehr oder weniger resorbiert werden, so ist doch über die Quantität des resorbierten Präparates gar keine Sicherheit vorhanden. Die namentlich bei größeren Dosen eintretenden Darmerscheinungen sind sogar sicherlich selbst die Ursache, daß recht viel von den eingenommenen Quecksilberpräparaten unverarbeitet mit den diarrhoischen Stühlen wieder herausbefördert wird. $\mathrm{Ob}$ dieser Verlust als ein erheblicher Schaden angesehen werden muß, ob nicht vielleicht schon die kleinsten resorbierten Mengen ausreichen, um eine ausreichende therapeutische Wirkung zu entfalten, bleibe dahin-

1) Welander, E. Einige Worte über die Form der Anwendung des Quecksilbers (Archiv für Dermatologie und Syphilis, Bd. XLVI). 


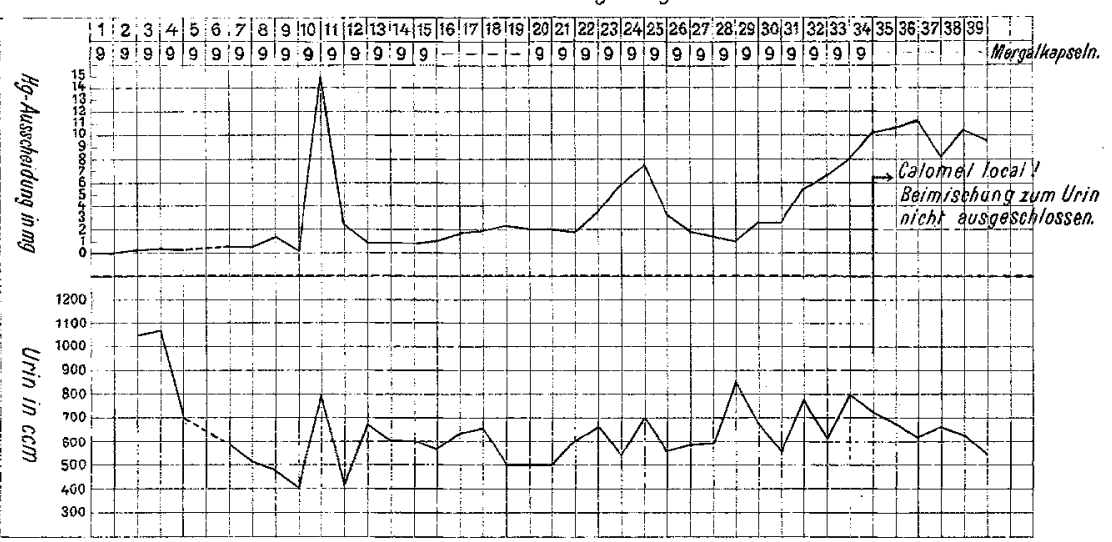

Curve 2

Behandlungstage

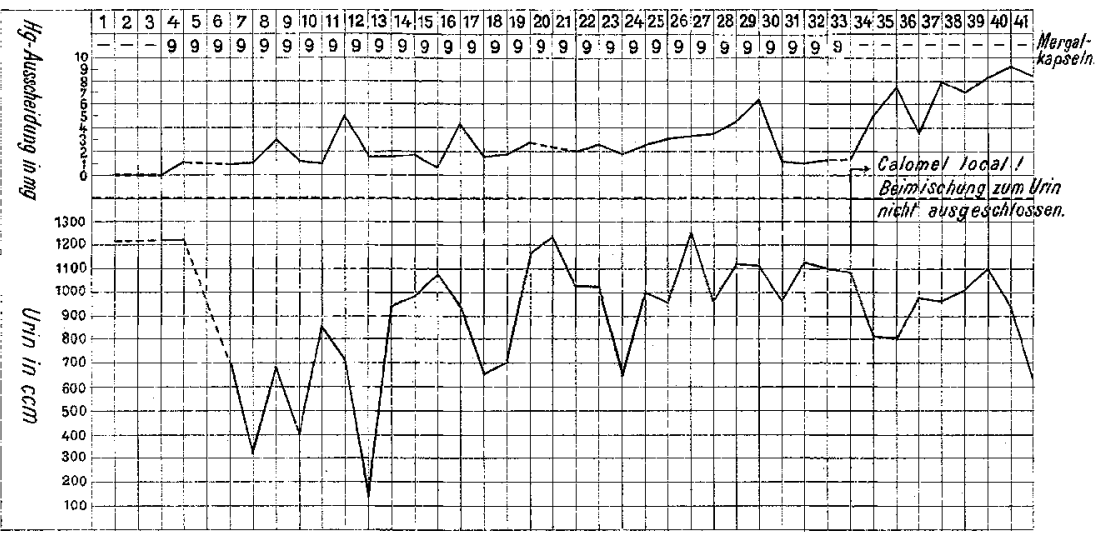

Curve 3

Behandlungstage

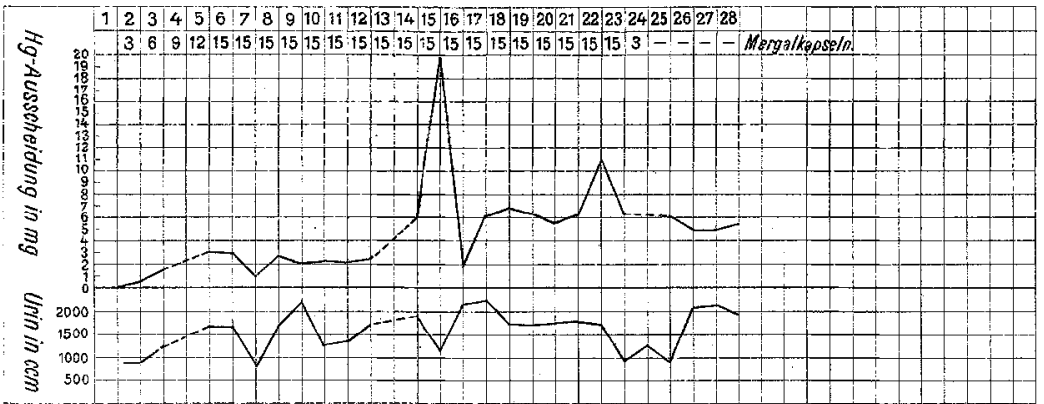

Höhne: Behandlung der Syphilis mit Mergal etc. 
gestellt." 1) Auch Neuma nn $n^{2}$ ) ist der Ausicht, daß von dem per os eingeführten $\mathrm{Hg}$ nur ein kleiner Teil zur Resorption gelangt, weshalb es nur langsam auf den syphilitischen Krankheitsprozeß einwirken und daher in schweren Fällen überhaupt keine Anwendung finden könne.

Trotzdem erscheint es berechtigt, immer wieder neue Quecksilberpräparate herzustellen und zu versuchen, $o b$ die interne als die bequemste und am meisten diskrete Bebandlung so modifiziert werden könne, daß sie einmal recht wirksam auf den syphilitischen Krankheitsprozeß sich erweist, dann auch frei von allen unangenehmen Nebenerscheinungen bleibt. Neuerdings ist nun von der Fabrik J. D. Riedel in Berlin ein Quecksilberpräparat unter dem Namen $\mathrm{M}$ e $\mathrm{r} \mathrm{g}$ al hergestellt worden und wird ron einer Reihe von Ärzten (B o s s, v. Zeissl, Keil, Leistikow, Saalfeld, Kanitz) zur internen Syphilisbehandlung empfohlen, z. T. allerdings mit gewissen Einschränkungen.

Mergal ist ein Präparat, das in Kapselform je 0.05 cholsaures Quecksilberoxyd + 0.1 Albuminum tannicum enthält. Das cholsaure Quecksilberoxyd hat nach Angabe von B os $\mathrm{s}^{3}$ ) einen $\mathrm{Hg}$-Gehalt von $23: 3 \%$.

In der hiesigen Klinik wurden im Laufe des letzten Jahres 43 Patienten mit Mergal behandelt. Hiervon litten 5 an primärer Syphilis mit regionären Drüsen, 30 an sekundärer, 1 an tertiärer Syphilis und 1 an beginnender Tabes. Von den 30 Fällen mit sekundärer Syphilis machten 26 die erste Kur überhaupt durch, hatten also noch nie $\mathrm{Hg}$ erhalten, einer die 4. Kur mit einem papulösen Exanthem, je einer die 2., bzw. 3. Kur ohne Erscheinungen. Secbs weitere Fälle hatten zu wenig Mergal erhalten, um in Betracht gezogen werden zu können, so daß für die Beurteilung nur 37, oder nach Abzug des Falles von Tabes, uur 36 in Frage kommen.

Bei der antisyphilitischen Kur wollen wir:

1. dem Körper möglichst große, genau bestimmbare Mengen ron Quecksilber einverleiben ohne jede oder wenigstens ohne jedewesentliche Belästigung des Kranken; gleichzeitig wollen wir:

1) Neisser, A. Die Einreibungskur. (Sammlung klinischer Vorträge, Nr. 199, Leipzig 1897.)

2) Neumann, J. Syphilis. Wien 1896.

s) Boss, S. Die Behandlung der Syphilis mit Mergal. Mediz. Klinik 1906. Nr. 30. 
2. die Erscheinungen, besonders die hochinfektiösen möglichst schnell beseitigen und so die Infektionsgefahr für andere herabdrücken; 3. Rezidive möglichst lange hinausschieben bzw. durch chronisch-intermittierende Behandlung (Fournier-Neisser) überhaupt unterdrücken. Sehen wir, wie sich die Behandlung mit Mergal diesen Forderungen gegenüber verhält.

1. Es erscheint theoretisch sehr leicht, dem Körper so viel wie möglich genau bestimmbare Mengen von Hg durch das Einnehmen von Mergalkapselnzuzufähren. Nur frägt es sich, wie viel von dem per os eingeführten Hydrargyrum vom Darmkanal wirklich resorbiert wird, und wieviel mit dem Kot, ohne zur Wirkung gekommenzu sein, den Körper verläßt. So viel steht jedenfalls fest, daß das Mergal, selbst in wesentlich höheren Dosen, als von den oben genannten Autoren angegeben wird - wir stiegen hier meist sehr schnell von 3 bis auf 15 Kapseln pro die (wie Saalfeld) - in der Mehrzahl der Fälle beschwerdelos vertragen wurde. Für die Schnelligkeit der Resorption ist das Erscheinen des $\mathrm{Hg}$ im Urin ein guter Indikator. Bei unseren Patienten war es 1 bis 6 Tage (einmal sogar erst 10 Tage) nach Beginn der Behandlung mit Mergal im Urin nachweisbar, meist nach 2 bis 3 Tagen. Diese Schwankungen in der Schnelligkeit der Hg-Ausscheidung deuten mit Wahrscheinlichkeit darauf hin, daß die Resorptionskraft des Darmes für Mergal individuell verschieden ist; womit auch die Ungleichmäßigkeit der Wirkung sich gut erklären würde. Auch die zwar selten, aber doch bisweilen beobachteten Durchfälle lassen den Schluß $\mathrm{zu}$, daß in solchen Fällen ein großer Teil des intern verabreichten $\mathrm{Hg}$ wirkungslos wieder ausgeschieden wird. Man ist also auch bei dieser Kur nicht ganz sicher, wie viel man in den erkrankten Organismus hineinbringt bzw. mit allen seinen Organen in Berührug bringt.

Nennenswerter Schaden oder Nachteil wurde hier trotz wochenlanger Zufuhr von 15 Mergalkapseln prodie niemals beobachtet. Sehr selten bestanden in den ersten Tagen der Kur Beschwerden von seitendes Magendarmkanals, die dann auch bald nachließen. Auch Durchfälle traten nicht häufig auf. Für den Fall, daß stärkere Magendarmstörungen sich einstellen, muß das Mergal ausgesetzt werden. Vielleicht Iassen sich aber diese Störungen durch Verwendung der Rumpelschen Kapseln (Capsulae geloduratae) vermeiden, die uns wenigstens bei der Anwendung des Sandelholzöles sehr gute Dienste geleistet haben. Eine stärkere Nierenreizung sahen wir im Gegensatz zu den anderen Autoren bei zwei poliklinischen Fällen (Eiweißausscheidung ohne Formelemente), bei deren einem das 
Mergal deswegen einige Zeit ausgesetzt werden mußte, außerdem noch in einem klinischen Falle. Auffallend war es, dab nur achtmal bei den verbältnismäßig hohen täglichen Dosen bis zu 15 Kapseln $(=0.75 \mathrm{Hg}$ cholicum oxydat. $=0.175 \mathrm{Hg}$ pro die wochenlang) eine leichte Stomatitis sich entwickelte (je 4 klinische und poliklinische Fälle). Nur einmal in einem klinischen Falle mußte das Mergal für wenige Tage ausgesetzt werden. Alle 8 Patienten hatten auffallend schlechte Zähne. Das legt die Vermutung nahe, daß nicht viel von dem eingeführten IIg wirklich vom Darmkanal resorbiert wird. Jedenfalls hatten wir nicht den Eindruck, daBselbstbei hohen Dosen eine Stomatitis leicht eintrat. "Wir werden um so mehr die GewiBheit, daß eine Kur wirklich eine gründliche Quecksilberwirkung erzielt habe, anstreben, je weniger wir einen unmittelbar sichtbaren Maßstab, ob diesor krfolg auch wirklich eingetreten sei, besitzen. Sobald die Syphilissymptome geschwunden sind, arbeiten wir eigentlich im Dunklen. Ob und welche therapeutischen Einwirlungen durch tortgesetzte Quecksilberzufuhr erzielt werden, wissen wir so wenig, daß wir sogar die meiner Überzeugung nach schädlichen Quecksilbernebenwirkungen manchmal nicht ungern anftreten sehen, weil wir damn wenigstens sicher wissen, daß überhaupt eine Quecksilbereinwirkung stattgefunden babe. " $(N \text { e iss er. })^{1}$ )

Die störungen, die wir überhaupt beobachtet haben, kamenabgesehenvon leichter Stomatitis tast a usschließlich bei poliklinischen Patientenvor und sind hier wohl durch mangelnde Sorgfalt z u erk lären; bei den klinischen Fällen führten, wie erwähnt, oft wochenlange Dosen von 15 Kapseln täglich nicht $z u$ den geringsten toxischen Erscheinungen.

2. Die Beeinflussung und Abbeilung der hochinfektiösen syphilitiscben Erscheinungen schien uns bisweilen recht langsam vor sich zu gehen, so dab wir in 5 Fällen gezwungen waren, nach 1 bis 2 Monaten zu Injektionen vou $\mathrm{Hg}$ salicyl. zu schreiten, die bald die gewürschte Wirkung entfalteten; 4 der Fälle sind in der Tabelle als $\mathrm{Nr} .2$ bis 4 und 9 enthalten. In 10 Fällen war die Wirkung des Mergals auf die vorhandenen Erscheinungen prompt und rasch und entsprach allen Anforderungen. Diesen Widerspruch scheint, wie bereits oben erwähnt, das individuell verschiedene Resorptionsvermögen des Darmes am leichtesten zu erklären. In 24 anderen Fällen war eine Beeinfussung der syphilitischen Prozesse unverkennbar, besonders die makulösen Exantheme zeigten bald eine Einwirkung, nur setzte die Abheilung, zumal der Schleimhautveränderungen, nicht mit der gewünschten Schnelligkeit ein, die wir bei Anwendung von Inunktions- oder Injektionskuren zu beobachten gewohnt sind. Es dürften aus diesem Grunde beim Bestehen hochinfektiöser Erscheinungen diese heiden Behandlungsmethoden der Mergalkur entschieden vorzuziehen sein, um die Infektionsgefahr für die Umgebung der Kranken so rasch wie möglich

1) Die Einreibungskur. (Sammlung klinischer Vorträge, Nr. 199.) 
einzuschränken. Ein tuberöses Syphilid warde durch eine 14 Tage lange, euergische Mergalkur nicht sehr beeinflußt. Ebenso war bei einem an Tabes dorsalis incipiens leidenden Patienten, bei dem frühere Lues zweifellos festgestellt war, trotz fünfwöchentlicher, sehr intensiver Mergalbehandlung keine deutliche objektive Besserung zu konstatieren, allerdings eine bedeutende im $\mathrm{subjektiven} \mathrm{Befinden.} \mathrm{Eine} \mathrm{direkte}$ Wirkung hatten wir auch nicht erwartet. Wir müssen daher Sa alfel $d^{1}$ ) vollständig beistimmen, der für die schweren Fälle, namentlich für solche, in denen ein lebenswichtiges Organ betroffen ist, vón der Mergalbehandlung abrät und die Injektionskur mit schwer löslichen Salzen empfiehlt. Betonen möchten wir nur, $d a \beta$ keiner unserer Fälle als ein schwerer zu bezeichnen war. Es handeite sich fast durchwegs um erste makulöse bzw. makulo-papulöse Exantheme der Haut und Schleimbäute. Lichenoide und ulzeröse Formen fehlten ganz, ebenso Palmar-und Plantarsyphilide.

3. Was die Schnelligkeit der Rezidive nach Mergalkuren anbetrifft, so ermöglicht die Kürze der Beobachtungszeit noch kein abschließendes Urteil. Bei einer Reihe von Patienten traten Rezidive schon während der Behandlung oder in den ersten 8 Wochen nach deren Beendigung auf. Um diese während der Behandlung neu auftretenden syphilitischen Erscheinungen und die Rezidive nach. Beendigung der Mergalkur besser zu veranschaulichen, stelle ich dieselben hier kurz in Form einer Tabelle zusammen. Vorausschicken will ich, daß sämtliche Patienten beim Beginn der Kur dentliche Erscheinungen der Sekundärperiode darboten, nur bei dreien, Nr. 4, 5 und 8, war das Exanthem im Anfang oder im Verlauf der Behandlung aufgetreten. Einen weiteren Fall, der währead und nach der Kur keine sekundären Erscheinungen darbot und 5 Wochen später mit nässenden Papeln in den Mundwinkeln und auf den Tonsillen wiederkam, habe ich nicht in die Tabelle aufgenommen. (Siehe umstehende Tabelle.)

Von 41 mit Mergal behandelten Patienten (23 in der Klinik, 18 in der Poliklinik) erkrankten als o 5 während der Kurmitfrischen syphilitischen Erscheinungen, 9 suchten bereits kurze Zeit ( 1 bis 2 Monate) nach Aussetzen der Behandlung wegen eines Rezidivs bei uns Hilfe (Fall 6 und 7 sind in jeder Gruppe gezählt). Dabei hatte die Mehrzahl dieser Fälle mehr als $15 \cdot 0$ cholsaures Quecksilber enthalten. Die im Verhältnis zu anderen Behandlungsmethoden recht hohe Zahl der schon während der Behandlung eingetretenen Rezidive scheint uns dagegen zu sprechen, daß man bei grundsätzlicher Ausdehnung der ersten

1) Saalfeld. Zur inneren Therapie del Syphilis. Therapeutische Monatshefte. 1907. Heft 1. 


\begin{tabular}{|c|c|c|c|c|}
\hline$\dot{\vec{z}}$ & $\mathrm{Na} \mathrm{me}$ & Ersehoinungen & Mergal & $\begin{array}{l}\text { Chlors. } \\
\text { Queck: } \\
\text { silber- } \\
\text { oxyd }\end{array}$ \\
\hline 1 & G. B. w. & $\begin{array}{l}\text { Keine Erscheinungen. } \\
\text { 2. Kur. }\end{array}$ & $\begin{array}{c}\text { In } 14 \text { Tagen } 100 \\
\text { Kapseln. }\end{array}$ & 50 \\
\hline 2 & V. L. w. & $\begin{array}{l}\text { Makul. Exanth. Plaques } \\
\text { auf den Tonsillen, breite } \\
\text { Kondylome. } \\
\text { 1. Kur. }\end{array}$ & $\begin{array}{c}\text { In } 33 \text { Tagen } 270 \\
\text { Kapseln. }\end{array}$ & 135 \\
\hline 3 & B. K. w. & 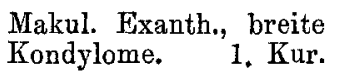 & $\begin{array}{c}\text { In } 30 \text { Tagen } 270 \\
\text { Kapseln. }\end{array}$ & $13 \cdot 5$ \\
\hline 4 & o. B. m. & $\begin{array}{l}\text { Primäraffekt, Drüsen, } 35 \\
\text { Tage spät. mak. Exanth. } \\
\text { Plaques muqu. 1. Kur. }\end{array}$ & $\begin{array}{c}\text { In } 65 \text { Tagen } 250 \\
\text { Kapseln. }\end{array}$ & $12 \cdot 5$ \\
\hline 5 & F. D. m. & $\begin{array}{l}\text { Primäraffekt, Drüsen, } 8 \\
\text { Tage später papulöses } \\
\text { Exanthem. }\end{array}$ & $\begin{array}{c}\text { In } 49 \text { Tagen } 300 \\
\text { Kapseln. }\end{array}$ & $15 \cdot 0$ \\
\hline 6 & A. D. m. & $\begin{array}{c}\text { Papulöses Exanthem. } \\
\text { 1. Kur. }\end{array}$ & $\begin{array}{l}\text { In } 101 \text { Tagen } \\
400 \text { Kapseln. }\end{array}$ & $20 \cdot 0$ \\
\hline 7 & M. F. m. & 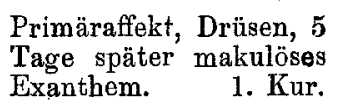 & $\begin{array}{l}\text { In } 27 \text { Tagen } 150 \\
\text { Kapseln, dann in } \\
\text { 64 Tag. } 250 \text { Kaps. }\end{array}$ & $20 \cdot 0$ \\
\hline 8 & P. H. m. & $\begin{array}{l}\text { Makulo-papulöses Exan- } \\
\text { them. } \\
\text { 1. Kur. }\end{array}$ & $\begin{array}{c}\text { In } 77 \text { Tagen } 350 \\
\text { Kapseln. }\end{array}$ & $17 \cdot 5$ \\
\hline 9 & R. K. m. & $\begin{array}{l}\text { Makulo-papulöses Exan- } \\
\text { them. }\end{array}$ & $\begin{array}{c}\text { In } 52 \text { Tagen } 370 \\
\text { Kapseln. }\end{array}$ & $18 \cdot 5$ \\
\hline 10 & F. K. m. & $\begin{array}{l}\text { Makulo-papulöses Exan- } \\
\text { them. }\end{array}$ & $\begin{array}{c}\text { In } 75 \text { Tagen } 300 \\
\text { Kapseln. }\end{array}$ & $15 \cdot 0$ \\
\hline 11 & E. Sp. m. & $\begin{array}{l}\text { Makulo-papulöses Exan- } \\
\text { them. }\end{array}$ & $\begin{array}{c}\text { In } 56 \text { Tagen } 400 \\
\text { Kapseln. }\end{array}$ & $20 \cdot 0$ \\
\hline 12 & R. Z. m. & 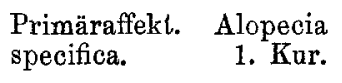 & $\begin{array}{l}\text { In } 2 \text { Monaten } \\
392 \text { Kapseln. }\end{array}$ & $19 \cdot 6$ \\
\hline 13 & P. G. m. & $\begin{array}{l}\text { Maculo-papulöses Exan- } \\
\text { them, Plaques muqu. auf } \\
\text { den Tonsillen. 1. Kur. }\end{array}$ & $\begin{array}{c}\text { In } 34 \text { Tagen } 350 \\
\text { Kapseln. }\end{array}$ & $17 \cdot 5$ \\
\hline 14 & E. F. m. & $\begin{array}{c}\text { Primäraffekt, makulo- } \\
\text { papulöses Exanthem. } \\
\text { 1. Kur. }\end{array}$ & $\begin{array}{c}\text { In } 52 \text { Tagen } 400 \\
\text { Kapseln. }\end{array}$ & $20 \cdot 0$ \\
\hline 15 & R. K. m. & $\begin{array}{c}\text { Primäraffekt, papulöses } \\
\text { Exanthem, Plaques mu- } \\
\text { queuses auf d: Tonsillen. } \\
\text { 1. Kur. }\end{array}$ & $\begin{array}{c}\text { In } 35 \text { Tagen } 322 \\
\text { Kapseln. }\end{array}$ & 16.1 \\
\hline
\end{tabular}




\begin{tabular}{|c|c|c|c|}
\hline \multicolumn{2}{|c|}{ Rezidiv } & \multirow{3}{*}{ Kein Einfluß } & \multirow{3}{*}{ Weitere Behandl. } \\
\hline während & nach & & \\
\hline \multicolumn{2}{|c|}{ der Behandlung } & & \\
\hline $\begin{array}{l}\text { Nach } 14 \text { Tag. näss. } \\
\text { Pap. ad genitalia. }\end{array}$ & - & - & Hg salicyl. \\
\hline - & $\begin{array}{l}\text { Nach } 21 / 2 \text { Woch } \\
\text { Plaques auf den } \\
\text { Tonsillen. }\end{array}$ & $\begin{array}{l}\text { Breite Kondyl. } \\
\text { am Schluß der } \\
\text { Behdl. unveränd. }\end{array}$ & $\begin{array}{l}\text { Kalomel lokal u. } \\
\text { Inj. }\left\{\begin{array}{c}\text { Hg salieyl. } \\
\text { Kalomel. }\end{array}\right.\end{array}$ \\
\hline- & - & $"$ & $\begin{array}{l}\text { Kalomel lokal, } \\
\mathrm{Hg} \text { salic.-Injekt. }\end{array}$ \\
\hline - & - & $\begin{array}{l}\text { Plaques muqu. der } \\
\text { Tonsillen bestehen } \\
\text { am SchluB der Boh. } \\
\text { noch (30 Tage nach } \\
\text { Auftreten d. Exant.). }\end{array}$ & $\mathrm{JK}$ \\
\hline - & $\begin{array}{l}\text { Nach 3 Wochen } \\
\text { circinäre Papeln } \\
\text { im Gesicht. }\end{array}$ & - & $\mathrm{Hg}$ salicyl. \\
\hline $\begin{array}{l}\text { Naeh } 20 \text { Tagen Plaqu. } \\
\text { muqu. an den Tons., } \\
\text { nach } 74 \text { T. a. d. Zunge. }\end{array}$ & $\begin{array}{l}\text { Nach } 7 \text { Wochen } \\
\text { cerebrale Syphilis } \\
\text { (Doppelsehen etc.) }\end{array}$ & - & Hg salicyl. \\
\hline $\begin{array}{l}\text { Nach } 3 \text { Wochen } \\
\text { Plaques muqueuses } \\
\text { der Tonsillen. }\end{array}$ & $\begin{array}{l}\text { Nach } 6 \text { Wochen } \\
\text { wieder Plaques } \\
\text { muqu. der Tons. }\end{array}$ & $\begin{array}{l}5 \text { Wochen lang } \\
\text { ausgesetzt wegen } \\
\text { st. Eiweißaussch. }\end{array}$ & - \\
\hline $\begin{array}{l}\text { Nach } 6 \text { u. } 7 \text { Woch. Auf- } \\
\text { schießen neuer Papeln, } \\
\text { nach } 10 \text { W.Pl.m. a.d.Ton. }\end{array}$ & - & - & Hg salicyl. \\
\hline- & - & $\begin{array}{l}\text { Nach } 52 \mathrm{~T} \text {. best. } \\
\text { das Exant. noch. }\end{array}$ & Hg salicyl. \\
\hline $\begin{array}{l}\text { Nach } 59 \text { Tagen (250 } \\
\text { Kap.) mak.-pap. Ex. }\end{array}$ & - & - & Weggeblieben. \\
\hline 一 & $\begin{array}{l}\text { Nach } 1 \text { Monat } \\
\text { Rezidivroseola. }\end{array}$ & - & - \\
\hline 一 & $\begin{array}{c}\text { Nach } 3 \text { W. Pl. m. } \\
\text { auf den Tons. }\end{array}$ & - & Oleum cinereum. \\
\hline - & $\begin{array}{c}\text { Nach 7 Wochen } \\
\text { papulo-krustöses } \\
\text { Exanthem. }\end{array}$ & - & Hg salicyl. \\
\hline- & $\begin{array}{l}\text { Nach } 1 \text { Monat } \\
\text { Plaques muqu. } \\
\text { auf den Tons. }\end{array}$ & - & Einreibungen. \\
\hline-- & $\begin{array}{c}8 \text { Tage spät. Tkterus. } \\
\text { starke Durchällle, } \\
\text { nach } 2 \text { Inj. Hg sal. } \\
\text { (zu h 1) fast beseitigt. }\end{array}$ & - & Hg salicyl. \\
\hline
\end{tabular}


Mergalkur auf 2 bis 3 Monate (Boss) wesentlich bessere Erfolge erreichen wird. Die Fälle 9 und 11 bis 15 hatten in weniger als 2 Monaten (in 34 bis 60 Tagen) 16.0 bis 20.0 cholsaures Quecksilberoxyd erhalten. Es scheint also die Mergalbehandlung keine sehr intensive und nachhaltige Wirkung auf den syphilitischen Krankheitsprozeß auszuüben. Neun von den in der Tabelle verzeichneten Fälle betrafen allerdings poliklinische Kranke, während die Erfolge bei klinischer Behandlung günstigere sind, hauptsächlich weil größere Dosen verwendet wurden und ohne Störung verwendet werden konnten. Mehr als 10 Mergalkapseln pro die vertrug jedenfalls nur ein Teil unserer poliklinischen Kranken und das auch nicht immer dauernd. Wir können also nach den hier gemachten Erfahrungen das Mergal für die erste Kur, die besonders energisch sein mub, nicht empfehlen, sondern müssen hierfür entschieden der Injektions- oder- Einreibungskur den Vorzug geben, die sicher eine viel intensivere und nachhaltigere Wirkung ausüben.

Das Mergal ibtaber andererseits zweifellos einen günstigen Einfluß auf syphilitische Veränderungen aus. Ob die nach unseren Erfahrungen dazu nötigen hohen Dosen bei poliklinischen Kranken sich durchführen lassen, scheint uns, soweit wenigstens unser Breslauer Krankenmaterial in Frage kommt; nicht sicher. Da aber auch der Verlauf bei unseren klinischen Patienten durchaus nicht immer ein sehr günstiger war, so ist es uns zweifelhaft, ob die Erfolge in der Privatpraxis, über die wir noch nicht berichten können, ähnlich gute sein werden, wie sie ron anderer Seite berichtet worden sind $\left(\mathrm{Kanitz}^{1}\right)$ sah unter 40 klinischen Fällen nur eiu Rezidiv während der Behandlung!). Jedenfalls ermöglicht das Mergal eine mildemeistbeschwerdelose und diskrete Behandlung der Syphilis, wird ab er von der Injektions- und Inunktionskur entschieden an Schnelligkeit und Sicherheit der

1) Kanitz, H. Über die interne Behandlung der Syphilis mit Mergal. Dermat. Zeitschr. 1907. Bd. XVI. Heft 7. 
Wirkung übertroffen. Bei allen floriden Erscheinungender Syphilis ist es unserer Erfahrung nach kontraindiziert (in $27.7 \%$ der Fälle (10 von 36 ) wirkte es allerdings, wie erwähnt, selbst für die erste Kur durchaus günstig), dagegen dürfte es sich, für die milden Zwischenkuren bei der chronisch-intermittierenden Behandlung wohl eignen, besonders wenn eine andere Kur nicht durchgefiihrt werden kann oder an Widerstand des Patienten scheitert. Da es das einzige in tern anzuwendende Hg-Präparat ist, das auch bei wochenlanger Anwendung nur selten Störungen hervorruft, die seine fernere Verwendung hindern, so kann es uns sehr unterstiutzen in dem Bestreben, bei jedem an Syphilis erkrankten Patienten die Behandlung chronischintermittierend durchzuführen, was sonst leider oft daran scheitert, daß andere Methoden den Patienten mehr belästigen oder in seinem Berufe stören.

Die außerordentliche Verschiedenheit der Erfolge bei klinischer Mergalanwendung hat uns veranlaßt, in einigen Fällen gleichzeitig Untersuchungen über die Ausscheidung des Quecksilbers durch den Harn vorzunehmen. Die qualitative Untersuchung nach Almén-Schillberg hatte, wie im klinischen Teil erwähnt ist, schon recht verschiedene Ergebnisse geliefert (Auftreten von $\mathrm{Hg}$ im Urin nach 1 bis 6 Tagen); es erschien deshalb erwünscht, auch quantitative Untersuchungen beizubringen. Über eingehende derartige Untersuchungen bei der Anwendung der verschiedenen Hg-Präparate haben vor kurzem erst in eingehender Weise $B \ddot{u}$ rgi $^{1}$ ) und Welander ${ }^{2}$ ) berichtet, auf deren Arbeiten wegen der allgemeinen Fragen verwiesen sei. Ebenso wie diese beiden Forscher haben wir uns, da es uns nur darauf ankam, Vergleichswerte. zu erhalten, darauf beschränkt, die tägliche Quecksilber-

1) Bürgi, E. Größe und Verlauf der Quecksilberausscheidung durch die Nieren bei den verschiedenen üblichen Kuren. Archiv für Dermat. Bd. LXXIX. 1906.

2) Welander, E. Zur Frage der Absonderung des Quecksilbers durch den Harn. Arch. f. Dermat. Bd. LXXXII. 1906. 
ausscheidung durch die Nieren zu überwachen. Die Ausscheidung durch den Kot konnte für.uns nicht in Frage kommen, da wir ein per os eingeführtes Präparat prüften. Es sei aber darauf hingewiesen, daß auch bei dieser Methode sicher resorbiertes Hg z. T. wieder durch den Darm ausgeschieden wird. Denn es ist keineswegs sicher, daß die Hauptmenge des resorbierten $\mathrm{Hg}$ durch die Nieren ausgeschieden. wird. Kronfeld und Stein ${ }^{1}$ ) sagen sogar, daß "die Ausscheidung durch den Darm der durch die Nieren nicht nachsteht." Andererseits wissen wir auch gar nicht, ob das Verhältnis der durch die Nieren und der durch den Darm abgesonderten Hg-Menge zu einander stets das gleiche ist, ob und in welcher Weise es schwankt, einmal von Tag zu Tag bei derselben Person, dann auch bei verschiedenen Kranken. Das erklärt vielleicht schon manche Unregelmäßigkeiten in der Hg-Ausscheidung durch die Nieren. bei unseren Fällen, ganz abgesehen davon, daß wir nichts darüber wissen, wie etwa die Resorptionsfähigkeit des Darmes bei verschiedenen Menschen und beim selben im Verlauf der Behandlung schwankt.

Für die quantitátiven Untersuchungen erfreuten wir uns der liebenswürdigen Unterstützung der Herren Prof. Dr. A begg und Privatdozent Dr. Herz vom hiesigen chemischen Institut der Universität, denen wir auch an dieser Stelle dafür unseren verbindlichsten Dank sagen. Unter ihrer Leitung hat Herr cand. chem. Willy Herrmann die Untersuchungen ausgeführt, dem wir dafür ebenfalls sehr zu Dank verpflichtet sind und der über die Methode folgendes berichtet: „Nach mebrfachen Versuchen mit den Methoden von Richards und Singer, Zenghelis und Farup, die zu keinem befriedigendem Resultate führten, habe ich mir folgende ansgearbeitet, die bei einer angewandten Menge 3 bis $4 \mathrm{mg}$ Quecksilber eine Fehlergreuze von 2 bis $3 \%$ ergeben bat. Der mit Salzsäure angesänerte Harn wird in einen geräumigen Rundkolben filtriert und mit Zinkstaub versetzt. Nach öfterem kräftigem Schütteln läßt man das gebildete Amalgam sich absetzen, gießt die über dem schweren Bodensatz stehende Flüssigkeit ab und dekantiert mit Wasser. Das Amalgam wird nun in Salzsäure gelöst, der allmählich Kaliumchlorat zugesetzt wird, was zur Vermeidung von Explosionen recht vorsichtig geschehen maß. Nach längerem Stehen ist das Amalgam gewöhnlich gelöst; war dies nicht vollstänđig der Fall, so wurde der Rundkolben mit einem langen Steigrohr versehen und erwärmt. Die Lösung wịrd alsdann in ein Becherglas gegossen, der Kolben mehrfach nachgespült und, um das Chlor zu vertreiben, die Flüssigkeit mit etwas Ammoniak

1) Zitiert nach Bürgi. 
versetzt. Um $\mathrm{zu}$ vermeiden, daß bei der nachherigen Behandlung mit Schwefelwasserstoff zu viel Schwefel ausgeschieden wird, muß das Chlor gänzlich verdrängt werden, was durch einen Kohlensäurestrom erreicht wird. Mit Schwefelwasserstoff wird nun ,das Quecksilber abgeschieden, das Sulfid in Goochtiegel abgesaugt, mit Wasser und Alkohol gewaschen, längere Zeit zur Entfernung von Schwefel mit Schwefelkohlenstoff behandelt und mit Alkohol und Äther nachgespült. Im Exsikkator wurde getrocknet und hernach gewogen."

Aus äußeren Gründen wurden die Versuche an zwei Frauen und einem Manne angestellt, die sämtlich an frischer bisher unbehandelter sekundärer Syphilis litten und noch nie $\mathrm{Hg}$ erhalten batten. Während der Dauer der Mergalanwendung wurde eine Lokalbehandlung mit Quecksilberpräparaten völlig unterlassen. Der Verlauf der Fälle ist aus den Tabellen und Kurven 1 bis 3 zu ersehen. (Siehe Tabelle 1-3.)

Übereinstimmend mit den Ergebnissen anderer Untersucher fand sich eine ziemliche Unregelmäßigkeit in der Ausscheidung bei Fall 1, wo mehrfach erhebliche Darmstörungen, ebenso wie eine zeitweise Eiweißausscheidung eintraten. In Fall 2 war die Ausscheidung eine mehr gleichmäßige. Leider war es in diesen beiden Fällen nicht zu erreichen gewesen, daß die Patientinnen sicher ihren gesamten Urin ablieferten. Dadurch sind die angegebenen, z. T. durchaus nicht kleinen Zahlen für die erste Zeit wohl etwas zu niedrig. Rechnet man für die ganz verloren gegangenen Mengen den Durchschnitt der Ausscheidung am roraufgegangenen und am folgenden Tage (unter "Bemerkungen" in Klammern beigefügt), so ergibt sich in Fall 1 bei 2411.55 mg Hg-Zufuhr (das cholsaure Quecksilberoxyd nach Boss mit einem Hg-Gehalt von $23.3 \%$ berechnet) eine Ausscheidung von $51 \cdot 2 \mathrm{mg}$ in $27 \mathrm{Tagen}^{1}$ ) also $2 \cdot 12 \%$ der eingeführten Menge. In Fall 2 betragen die gleichen Zahlen $2831 \mathrm{mg} \mathrm{Hg}$ mit einer Ausscheidung durch die Nieren von $61.95 \mathrm{mg} \mathrm{Hg}=2 \cdot 18 \%$ der eingeführten Menge. In beiden Fällen trat die Abheilung der Symptome nur langsam und ungenügend ein. $\mathrm{Ob}$ das darauf beruht, daß nur ein so geringer Prozentsatz der eingeführten Menge verarbeitet wurde (Darmstörungen!) oder ob die Aufnahmefähigkeit des Darmes

1) Diese Zeit wurde gewählt, weil Fall 3 nur 27 Tage untersucht werden konnte. Auf 33 Tage berechnet, würde sich das Verhältnis für Fall 1 etwas günstiger stellen: $3040^{\circ} 65 \mathrm{mg}$ Zufuhr mit $77^{\circ} 15 \mathrm{mg}$ Ausscheidung $=2.53 \%$. 
Tabelle I.

Tabelle II.

V. L., 19 J., Oedema ind., mak. Ex., Plaqu. $\quad$ B. K, 24 J., makulöses Exanthem, nässendo muqu. auf d. Tons., näss. Pap. an den Gen. Papeln an den Genitalien.

\begin{tabular}{|c|c|c|c|c|c|c|c|c|}
\hline 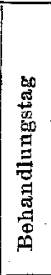 & 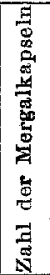 & 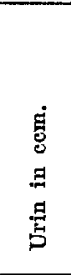 & 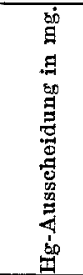 & Bemerkungen & 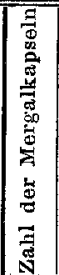 & 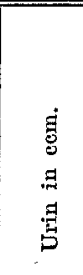 & 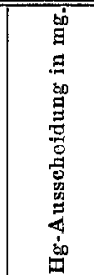 & Bemerkungen \\
\hline 1. & 9 & - & - & & - & - & - & \\
\hline 2. & 9 & 1045 & 02 & & - & - & - & \\
\hline 3. & 9 & 1065 & 0.5 & & -1 & - & - & \\
\hline 4. & 9 & 700 & 0.3 & Schwitzen & 9 & 1225 & $1 \cdot 0$ & \\
\hline 5. & 9 & - & - & $(0 \cdot 4)$ & 9 & - & - & $(0.9)$ \\
\hline 6. & 9 & 595 & 0.5 & & 9 & 700 & 0.8 & \\
\hline 7. & 9 & 510 & 0.5 & $=0.05$ & 9 & 325 & $1 \cdot 0$ & Schwitzen. \\
\hline 8. & 9 & 480 & 1.5 & $\Leftrightarrow$ Schw. & 9 & 680 & $3 \cdot 0$ & \\
\hline 9. & 9 & 400 & 02 & 层泀 & 9 & 400 & $1 \cdot 4$ & Snur Fiweiß \\
\hline 10. & 9 & 800 & 150 & $1+5$ & 9 & 840 & 1.0 & opur nawerb. \\
\hline 11. & 9 & 425 & $2 \cdot 4$ & Appetit dau- & 9 & 710 & $4 \cdot 7$ & Schwitzen. \\
\hline 12. & 9 & 670 & 0.9 & ernd sehlecht to & 9 & 135 & 1.5 & \\
\hline 13. & 9 & 600 & $0 * 9$ & & 9 & 940 & 1.5 & \\
\hline 14. & 9 & 565 & $1 \cdot 1$ & & 9 & 985 & $1 \cdot 6$ & \\
\hline 15. & 9 & 625 & $1 \cdot 8$ & Sehr starke Leib- & 9 & 1075 & 06 & \\
\hline 16. & - & 625 & 1.9 & $\begin{array}{l}\text { sehmerzen, keine } \\
\text { Diarrhöen. }\end{array}$ & 9 & 930 & $4 \cdot 3$ & Schwitzen. \\
\hline 17. & - & 650 & 1.9 & sehwitzen. & 9 & 650 & $1 \cdot 7$ & \\
\hline 18. & - & 500 & $2 \cdot 2$ & & 9 & 700 & 1.8 & \\
\hline 19. & - & 500 & $2 \cdot 0$ & & 9 & 1165 & $2 \cdot 6$ & \\
\hline 20. & 9 & 500 & $2 \cdot 0$ & Exanth. geschw. & 9 & 124.0 & Verlust & $(2 \cdot 3)$ \\
\hline 21. & 9 & 600 & 1.7 & Schwitzen. & 9 & 1025 & $2 \cdot 0$ & Schwitzen. \\
\hline 22. & 9 & 650 & $3 \cdot 5$ & & 9 & 1020 & $2 \cdot 6$ & \\
\hline 23. & 9 & 530 & $5 \cdot 7$ & & 9 & 640 & $1 \cdot 85$ & \\
\hline 24. & 9 & 695 & $7 \cdot 5$ & Schwitzen. & 9 & 1000 & $2 \cdot 45$ & Schwitzen. \\
\hline 25. & 9 & 560 & $3 \cdot 4$ & & 9 & 960 & $2 \cdot 9$ & \\
\hline 26. & 9 & 580 & $1 \cdot 8$ & & 9 & 1250 & $3 \cdot 2$ & \\
\hline 27. & 9 & 590 & Verlust & $(1 \cdot 4)$ & 9 & 955 & $3 \cdot 4$ & Exanth. geschw. \\
\hline 28. & 9 & 850 & 1.0 & Schwitzen. & 9 & 1120 & $4 \cdot 45$ & Schwitzen. \\
\hline 29. & 9 & $6 £ 0$ & $2 \cdot 5$ & & 9 & 1110 & $6 \cdot 2$ & \\
\hline 30. & 9 . & 550 & $2 \cdot 45$ & & 9 & 960 & $1 \cdot \overline{2}$ & \\
\hline 31. & 9 & 770 & $5 \cdot 5$ & Schwitzen: & 9 & 1125 & 0.9 & Schwitzen. \\
\hline 32. & 9 & 610 & 6.5 & & 9 & 1100 & Verlust & $(1 \cdot 65)$ \\
\hline 33. & 9 & 800 & 80 & & 9 & 1080 & 245 & Cond. lata unver- \\
\hline 34. & 9 & 725 & $10 \cdot 1$ & Condylom. lata & - & 805 & $4 \cdot 9$ & ändert. \\
\hline 35. & - & 670 & $10 \cdot 6$ & bestehen noch. & - & 800 & $7 \cdot 5$ & Urin nicht mit \\
\hline 36 & - & 620 & $11 \cdot 2$ & Urin nicht mit & - & 980 & 37 & dem Katheter \\
\hline 37. & - & 655 & $8 \cdot 2$ & dem Katheter & - & 960 & $8 \cdot 1$ & entnommen. \\
\hline 38. & - & 625 & $10 \cdot 4$ & entn. Kal. auf & - & 1005 & $7 \cdot 0$ & Kalomel auf \\
\hline 39. & - & 545 & $9 \cdot 6$ & die Cond. lata. & - & 1100 & $8 \cdot 2$ & die Condylo- \\
\hline 40. & - & - & - & & - & 940 & $9 \cdot 1$ & mata lata. \\
\hline 41. & - & - & 一 & $\mid \begin{array}{c}\text { In } 27 \mathrm{Tag} \text {. eing. } \\
2416 \mathrm{mg} \text {. Hg, } \\
\text { ausg. } 51 \cdot 2 \mathrm{mg} . \mathrm{Hg} \\
\quad=2.12 \%\end{array}$ & - & 640 & $8 \cdot 4$ & $\begin{array}{l}\text { In 27 Tagen eingef. } \\
2831 \mathrm{mg} . \mathrm{Hg} \text {, ausge- } \\
\text { schieden 61.95 mg. } \\
\text { sg =2.18 Prozent. }\end{array}$ \\
\hline
\end{tabular}


Tabelle III.

K. F., 31 J., Ulcera dura, papalöses Exanth., Plaques muqueuses auf den Tonsillen.

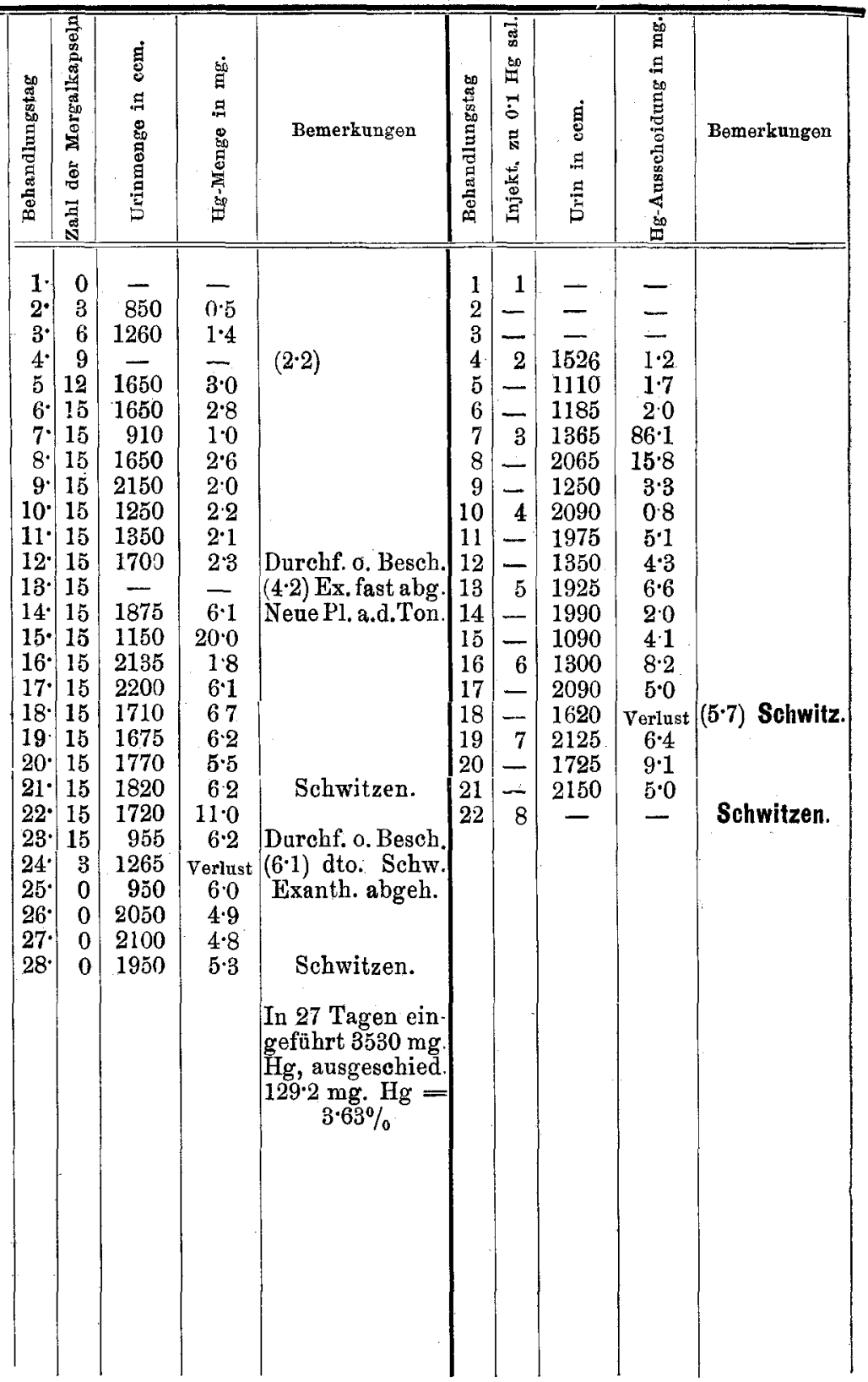

Tabelle IV.

P. K., 29 J., papulo-pustulöses Exanthem. $\mathrm{Hg}$ salicyl-Injektionon. 
bei beiden Patientinnen eine geringe war, vermögen wir nicht zu sagen. Bei der geringen Heilungstendenz scheint uns zur Erklärung nicht zu genügen, daß zuweilen nur ein Teil des Urins zur Untersuchung gekommen ist. Günstiger liegen die Verhältnisse für Fall 3. In 27 Tagen wurde bei Einführung von $3530 \mathrm{mg} \mathrm{Hg} \mathrm{129.2} \mathrm{mg} \mathrm{Hg}$ durch die Nieren ausgeschieden, also $3.63 \%$. Das prompte Abheilen der Erscheinungen (allerdings Auftreten neuer Plaques muqueuses auf den Tonsillen am 16. Tage) ist hier vielleicht Folge einer besseren Aufnahmefähigkeit der Darmschleimhaut. In Fall 1 und 3, weniger in Fall $2 \mathrm{nahm}$ die $\mathrm{Hg}$-Ausscheidung gegen das Ende der Behandlung etwas zu. Vielleicht darf man daraus schließen, daß durch lange Zeit fortgesetzte Mergalbehandlung die Wirkung eine günstigere wird. In Fall 1 und 2 sind die Zahlen vom 34. Tage $a b$ allerdings nicht verwertbar, da eine Kalomelbeimischung zum Urin nicht auszuschließen ist.

Abgesehen ron ihrer Ungleichmäligkeit ist die Hg-Ausscheidung durchaus keine ganz geringe. Wir sehen sogar Tagesdosen von $15 \mathrm{mg}$ (Fall 1) und $20 \mathrm{mg}$ (Fall 3), also etwa den sechsten Teil der an einem Tage eingeführten $\mathrm{Hg}$-Menge, während die Mehrzahl der früheren Untersucher bei interner Verabreichung von Hg-Präparaten mit Ausnahme von $B$ ürgi nur erheblich kleinere Dosen gefunden haben. Diese auffallende Beobachtung veranlabte uns, einige Zeitlang auch die Hg-Ausscheidung bei einem Patienten zu untersuchen, der Injektionen von $\mathrm{Hg}$ salicyl. (jeden vierten Tag $0 \cdot 1$ ) erhielt. Der Patient hatte vor längerer Zeit eine Schmierkur durchgemacht und hatte vor Beginn der Untersuchung schon eine Injektion erhalten. Dadurch sind die Zahlen möglicherweise etwas höher als wir sie sonst erhalten hätten. Die tägliche Hg-Ausscheidung schwankte erheblich, nahm aber im allgemeinen allmählich zu und ist bedeutend höher als bei Mergalanwendung. Auch hier trat einmal an zwei aufeinanderfolgenden Tagen eine außerordentlich starke Ausscheidung auf $\left(86^{\circ} 1 \mathrm{bzw}\right.$. $15.8 \mathrm{mg}$ !), für die wir Untersuchungsfehler nicht verantwortlich machen können. Es war nur auffällig gewesen, dab an den vorhergehenden Tagen die Hg-Absonderung eine sehr geringe gewesen war (siehe Tab. 4). 
Es scheint so nicht ausgeschlossen, daß eine Massenresorption zu einer Massenausscheidung von $\mathrm{Hg}$ durch die Nieren geführt hat, die am Tage der dritten Injektion sogar die eingeführte $\mathrm{Hg} \cdot$ Menge um fast die Hälfte übertroffen hat (Hg. salicyl. entbält $59 \% \mathrm{Hg}$ ). Diese ähnlichen Vorkommnisse bei ganz verschiedenen Kranken und zu ganz verschiedenen Zeiten sprechen wohl dagegen, daß hier "artefizielle“" Wirkungen vorliegen. Trotz genauester Nachforschung hat sich auch keine Unregelmäßigkeit feststellen lassen. Daß die Ausscheidung am Injektionstage hier durchaus nicht immer am stärksten war (B ürgi, W elander), ist vielleicht durch das Suspensionsmittel (Oleum Dericini) bedingt. Irgendwelche weitergehenden Schlüsse können wir aus dieser einęı Versuchsreibe natürlich nicht ziehen. (Siehe Tabelle 4.)

Ein Einfluß des Schwitzens auf die $\mathrm{Hg}$-Absonderung ist aus unseren Tabellen nicht zu ersehen. $\mathrm{Ob}$ die Hg-Ausscheidung durch den Schweiß überhaupt nur eine minimale ist, vermögen wir daher nicht $\mathrm{zu}$ beurteilen; dazu wäre eine gesonderte Untersuchung des gewonnenen Schweißes nötig.

In unseren Versuchen war der Prozentsatz des durch die Nieren abgesonderten $\mathrm{Hg}$ im Verhältnis zur eingeführten Menge recht gering, höchstens $3.63 \%$ für den sehr günstig beeinflußten Fall 3. Bürgi sah bei Anwendung von Hydrargyr. jodatum flavum $7 \cdot 52 \%$ und von Kalomel $7 \cdot 11 \%$ der eingeführten Menge im Urin erscheinen. Vielleicht erklärt aber das die Möglichkeit, $\mathrm{da} B$ dem Organismus so große Mengen $\mathrm{Hg}$ in der Form des Mergals zugeführt werden können, ohne daß Störungen auftreten, wie bei anderen innerlich angewendeten Mitteln.

Leider konnte aus äußeren Gründen (Entlassung der Patienten) die Untersuchung nicht längere Zeit nach Aussetzen der Mergalbehandlung fortgesetzt werden. Wir haben also an unseren Fällen Untersuchungen über die Remanenz des $\mathrm{Hg}$ im Organismus nicht anstellen können. Die Häufigkeit der frühen Rezidive, die wir bei unseren Patienten gesehen haben, ist vielleicht dadurch zu erklären, daß vom cholsauren Quecksilberoxyd zwar nicht unbedeutende Mengen resorbiert werden, daß aber das Mittel sehr schnell wieder ausgeschieden wird, während bei anderen Behandlungsmethoden das $\mathrm{Hg}$ monatelang. 
im Körper bleibt. Ob jene resorbierten Mengen genügende sind, vermögen wir nicht zu sagen, da wir über die geringste Menge zur Syphilisbehandlung nötigen Quecksilber überhaupt im Unklaren sind. Nach unseren klinischen Erfahrungen ist das aber für die erste Kur jedenfalls nicht der Fall oder wenigstens nur ausnahmsweise.

Gehen wir ron dem Gesichtspunkt aus, daß die Wirkung eines Mittels im Verhältnis zu der in den Körper eingefïhrten Menge steht, so ergeben auch die quantitativen Untersuchungen eine Bestätigung unserer klinischen Erfahrungen, daß bei hohen Dosen (15 Kapseln pro die) nicht nur bessere Erfolge erzielt werden, sondern auch die Hg-Absonderung durch die Nieren eine größere ist. Im allgemeinen dürfen wir wohl annehmen, daß einer größeren Resorption auch eine größere Ausscheidung entspricht, wenn auch beide durchaus nicht immer Hand in Hand gehen.

Wir sind uns natürlich völlig klar, daß unsere wenigen Untersuchungsreihen ein abschließendes Urteil nicht erlauben. Wir glauben aber mit diesem Vorbehalt aus unseren Versuchen über die Anwendung des Mergals folgendes schließen zu können:

Die Hg-Ausscheidung durch die Nieren ist keine unbedeutende, aber nicht sehr regelmäßig, doch scheint sie allmählich zuzunehmen. Abhängig ist sie sicher ron individuellen Verschiedenheiten der Resorption. Eine mangelhafte Abheilung der klinischen Erscheinungen scheint auf eine geringe Resorptionsgröße hinzudeuten, wie in Fall 1 und 2.

Da auch beim Gebrauch des Mergals, wie bei innerlicher Anwendung anderer Hg-Präparatedas Verbleiben des Hg im Organismus nur ein geringes ist, so ist das Präparat fürenergischeKuren nicht zu empfehlen, kann aber, wie wir schon im klinischen Teil ausgeführt haben, wohl mit Vorteil für die milden Latenzkuren bei chronisch-intermittierender Behandlung der Syphilis verwendet werden.

Ich möchte nicht verfehlen, Herrn Oberarzt Dr. Zieler für die mannigfache Hilfe bei der Anfertigung der Arbeit auch an dieser Stelle meinen besten Dank zu sagen. 